\title{
Controllable phycobilin modification: an alternative photoacclimation response in cryptophyte algae
}

\author{
Leah C. Spangler ${ }^{1}$, Mina Yu ${ }^{1}$, Philip D. Jeffrey ${ }^{2}$ and Gregory D. Scholes ${ }^{1 *}$ \\ (1) Department of Chemistry, Princeton University; (2) Department of Molecular Biology, \\ Princeton University.
}

\begin{abstract}
Cryptophyte algae are well known for their ability to survive under low light conditions through the use of their auxiliary light harvesting antennas, phycobiliproteins. Mainly acting to absorb light where chlorophyll cannot $(500-650 \mathrm{~nm})$, phycobiliproteins also play an instrumental role in helping cryptophyte algae respond to changes in light intensity through the process of photoacclimation. Until recently, photoacclimation in cryptophyte algae was only observed as a change in the cellular concentration of phycobiliproteins; however, an additional photoacclimation response was recently discovered that causes shifts in the phycobiliprotein absorbance peaks following growth under red, blue, or green light. Here, we reproduce this newly identified photoacclimation response in two other species of cryptophyte algae, $P$. sulcata and $H$. pacifica, and elucidate the origin of the response on the protein level. We compare isolated native and photoacclimated phycobiliproteins for these two species using spectroscopy and mass spectrometry, and we report the x-ray structures of the PC577 light harvesting complex and corresponding photoacclimated complex. We find that neither the protein sequences, nor the protein structures are modified by photoacclimation. We conclude that cryptophyte algae change a chromophore in one site of their phycobiliprotein $\beta$ subunits as part of the photoacclimation response to changes in the spectral quality of light. Ultrafast pump-probe spectroscopy shows that the energy transfer is weakly affected by the photoacclimation.
\end{abstract}

\section{Synopsis}

An alternative photoacclimation response in cryptophyte algae produces phycobiliproteins with modified absorbance, which was found to originate from the modification of bilin chromophore identities within the unchanged apoprotein structure. 


\section{Introduction}

Photoacclimation is a process performed by photosynthetic organisms that is used to regulate their internal energy production following a change in light intensity. ${ }^{1}$ For example, a decrease in light intensity will trigger a photosynthetic organism to increase its intracellular chlorophyll concentration, allowing each cell to harvest more light in order to compensate for the loss of incident light. In addition to compensating for overall changes in light intensity, photoacclimation can also be used to offset the loss of light in a specific wavelength region. This unique type of photoacclimation, known as chromatic acclimation, only occurs in photosynthetic algae which contain auxiliary light harvesting antennae known as phycobilisomes - large protein superstructures capable of harvesting light in a region where chlorophyll cannot: $500-650 \mathrm{~nm}^{2}$ Phycobilisomes are primarily found in algae which live deep in the ocean, such as cyanobacteria and red algae, and thus require the ability to harvest light across the entire solar spectrum for survival. ${ }^{3,4}$ Because of the highly crowded and dynamic nature of their aqueous environments, these algae species utilize chromatic acclimation to quickly compensate for changes to the available wavelength regions of light. ${ }^{5}$ This photoacclimation strategy occurs quickly, often in as little as seven days, and allows cyanobacteria to quickly acclimate to fluctuations in visible and far-red light. ${ }^{6,7}$ Chromatic acclimation has recently been shown to occur in $\sim 40 \%$ of cyanobacteria across the globe, and is found in both freshwater and ocean environments. ${ }^{8,9}$

The most commonly observed mechanism for chromatic acclimation used by cyanobacteria and red algae is the modification of the phycobilisome. ${ }^{2}$ Each phycobilisome is made up of two or more types of phycobiliproteins, each with a unique absorbance region resulting from the eight tetrapyrrole bilin chromophores housed within the apoprotein structure. Each type of phycobiliprotein contributes to the overall absorbance spectra of the phycobilisome and can therefore be used to control the resultant absorbance spectra used for light harvesting. To date, researchers have identified six types of chromatic acclimation in cyanobacteria; four types are used to acclimate to changes of light in the visible region. ${ }^{10}$ Types 1 and 2 involve phycobilisomes with only one type of phycobiliprotein. Acclimation is achieved by addition, removal, or detachment of the phycobiliproteins from the rod-like structure of the phycobilisome. Type 3 is uses substitution of the individual protein building blocks of their phycobilisome superstructure. ${ }^{11}$ A recently discovered strategy, type 4 chromatic acclimation, modifies the individual chromophores of the phycobiliproteins, which remain attached to the phycobilisome. ${ }^{12}$

Because the mechanism for chromatic acclimation requires the phycobilisome superstructure, this specialized photoacclimation response was previously believed to occur only in cyanobacteria and red algae. ${ }^{10}$ However, a similar photoacclimation response was recently observed in a species which descended from red algae but completely lacks a phycobilisome: cryptophyte algae. ${ }^{13}$ While cryptophytes no longer contain a phycobilisome, each species has retained the ability to express phycobiliproteins, which are instead packed into the thylakoid lumen of the chloroplasts without any sort of structural arrangement. ${ }^{14}$ Additionally, each cryptophyte species is only capable of expressing one type of phycobiliprotein, meaning cryptophytes should 
no longer be able to photoacclimate to changes in spectral quality. ${ }^{15}$ However, the cryptophyte algae Hemiselmis cryptochromatica was recently discovered to have shifted phycobiliprotein absorption peaks following growth under red, green, or blue light. ${ }^{13}$ This change in phycobiliprotein absorbance is surprising - how are cryptophytes, who lack a structured phycobilisome and variation in phycobiliproteins, able to produce absorption peak shifts in their phycobiliproteins?

To address this question, we consider the origin of the phycobiliprotein absorbance peak. In cryptophytes, there are eight unique types of phycobiliproteins which range from 545 to 645 $\mathrm{nm}$ in peak absorbance, and these spectral differences result from not only the chemistry of the specific chromophores contained in each phycobiliprotein but also from the interactions between the chromophores and their protein surroundings. ${ }^{16,17}$ The strong dependence of the absorbance spectra on each protein's chromophore composition and apoprotein structure suggests two potential methods for cryptophyte photoacclimation: a modification to the chromophore identities within the phycobiliprotein, or a subtle alteration to the apoprotein structure. The former hypothesis is supported by the existence of the previously discussed type 4 chromatic acclimation, recent discovered in the cyanobacteria strain Synechococcus, which does not swap out entire phycobiliproteins in its phycobilisome but instead modifies the individual bilin chromophores of each phycobiliprotein, preserving the original protein structure. ${ }^{18-20}$ This new acclimation mechanism, also known as differential chromophorylation, may be retained in cryptophyte algae, preserving their ability to photoacclimate following changes in spectral quality.

In this work, we reproduced this newly discovered photoacclimation response in two other species of cryptophyte algae, Proteomonas sulcata and Hemiselmis pacifica, and used both spectroscopic and biophysical measurements to uncover the origin of the phycobiliprotein absorbance shifts on a biological level. We found that both of the cryptophyte species shifted their absorption spectra following growth under restrictive light conditions. Importantly, we confirmed that the absorbance shifts originated from the individual phycobiliproteins and not the cellular environment by isolating the phycobiliproteins from the cell. Analysis of the phycobiliprotein structure using mass spectroscopy, CD measurements, and x-ray crystallography confirmed that there was no change in amino acid sequence or a structural perturbation which could cause an absorbance peak shift. Instead, we found that cryptophytes appear to have retained the ability to photoacclimate though differential chromphorylation of their constituent bilin chromophores. We found that a change in the bilin chromophore did not affect the ultrafast dynamics of energy transfer in the photoacclimated phycobiliproteins, indicating that the protein structure continues to play a dominant role in energy transfer. 


\section{Results}

In this work, we chose to study the photoacclimation response of two species of cryptophyte algae, which differ in genus and phycobiliprotein production. The first species, Proteomonas sulcata, contains phycoerythrin 545 (PE545), which harvests light in the 520-580 $\mathrm{nm}$ region with the major peak at $545 \mathrm{~nm}$ (Figure 1a). The second species, Hemiselmis pacifica contains phycocyanin 577 (PC577) which absorbs light in the $550-620 \mathrm{~nm}$ region with a major peak at $577 \mathrm{~nm}$ (Figure 1b). The light absorbance of each phycobiliprotein can be identified in the absorbance spectra of the whole cell algae, Figure $1 \mathrm{c}, \mathrm{d}$, as peaks that correspond to the isolated absorbance of the individual phycobiliproteins. In addition to peaks from the corresponding phycobiliproteins, each whole cell absorbance spectra also contains peaks at 435 and $680 \mathrm{~nm}$, resulting from chlorophyll containing light harvesting complexes and photosystems, used by cryptophytes for addition light harvesting and photosynthesis. Each spectrum also contains a peak around $500 \mathrm{~nm}$, which originates from the carotenoid alloxanthin - these are auxiliary pigments which provide some additional light harvesting but are mainly believed to be used for dissipating excess energy under high light conditions to prevent photooxidation which leads to cell death. ${ }^{21}$

Phycobiliprotein light absorption is essential for cryptophyte energy production and survival. When the amount of available light is reduced or restricted, the algae maintain cellular energy production through the process of photoacclimation. ${ }^{22}$ To examine the photoacclimation response in cryptophyte algae, we restricted the available light spectra during cryptophyte growth using filters and monitored the effect on the phycobiliproteins using absorbance spectroscopy. The light transmitted through the filter, shown as the shaded overlay in Figure $1 \mathrm{c}$,d, only slightly overlaps the absorbance region of the phycobiliprotein in each species. We matched the overall light intensity for both white light and restricted light cultures to avoid changes caused by the quantity of photosynthetically active light. We monitored the growth of each culture using daily cell counts to ensure the growth rate was unaffected (SI, Figure S1), and probed the algae for a photoacclimation response by comparing the absorbance spectra for cultures grown with and without restricted light conditions throughout the growth period.

After two weeks of growth, both $P$. sulcata and H. pacifica grown under light restricted conditions showed an increase in the intensity of the absorbance peak corresponding to the phycobiliprotein (Figure $1 \mathrm{c}, \mathrm{d}$ ). The absorbance spectra were normalized by cell concentration ( Figure S1), so the absorbance intensity corresponds to the phycobiliprotein concentration per cell. Thus, each species demonstrated an increase in phycobiliprotein concentration per cell. The upregulation of phycobiliprotein production was expected; this is a common photoacclimation response and was previously observed in $P$. sulcata grown under low light conditions. ${ }^{23}$ The very minor change in the chlorophyll containing LHCs and photosystems is also consistent with previous reports of cryptophyte photoacclimation, which show only regulation of the phycobiliprotein concentration. ${ }^{13}$ The absorbance peak intensity corresponding to the carotenoid alloxanthin was also unaffected, which was surprising given that the absorbance peak lies within 
the region of available light. The minimal change in carotenoid absorbance intensity supports previous observations that carotenoids do not play a significant role in light harvesting. ${ }^{24}$

Closer examination of the whole cell absorbance spectra in the phycobiliprotein region shows an additional, more subtle photoacclimation response. We normalized the whole cell absorbance spectra by the relative center of the absorbance peak corresponding to the phycobiliprotein (557 nm for PE545, and $600 \mathrm{~nm}$ for PC577) (Figure $1 \mathrm{e}, \mathrm{f}$ ) to more clearly illustrate the change. The absorbance peak corresponding to PC577 in H. pacifica (Figure 1f) shows a significant blue shift of the major peak at $577 \mathrm{~nm}$ by about $5 \mathrm{~nm}$, and a decrease in the shoulder of the absorbance peak at $620 \mathrm{~nm}$. Both of these changes were consistent with the newly available light spectrum under restricted conditions. In contrast, $P$. sulcata did not show a significant blue shift in absorbance; the small blue shift in the PE545 absorbance peak (Figure 1e) was not found to be statistically significant when measured across three independent cultures.

The change in absorbance to the phycobiliprotein region in $H$. pacifica could have originated in two ways: a modification to the phycobiliprotein itself, or an environmentally induced perturbation to the protein caused by the cell. To determine whether the change in absorbance originated from variations in the cellular environment, we extracted the phycobiliproteins from cultures grown under full and restricted light conditions and subsequently measured the absorbance spectra of the purified proteins. We found that PC577 harvested from $H$. pacifica grown under restricted light conditions maintains a blue shift in absorbance, Figure $1 \mathrm{~h}$, confirming that the change is not a result of the cellular environment but indeed a modification to the phycobiliprotein itself. We also measured the fluorescence spectra of the purified PC577 and found no observable changes. Phycobiliprotein fluorescence mainly originates from the lowest energy population of states, indicating that the final energy transfer step out of the phycobiliprotein remained unchanged. ${ }^{25}$

Surprisingly, the absorbance spectrum of PE545 was also modified once extracted from the cellular environment, despite there being no observable change in the whole cell absorbance spectra (Figure 1g). The absorbance spectrum of purified PE545 from restricted light conditions had an enhanced shoulder at $570 \mathrm{~nm}$ and a slightly broader absorbance spectra when compared to PE545 extracted from algae grown under full light. We also observed the disappearance of a secondary peak at $650 \mathrm{~nm}$, which was not studied in detail but may result from a secondary population of phycobiliprotein which is only expressed under normal light conditions. The fluorescence spectra of PE545 (Figure 1g) show no change as a result of growth under restricted light, in agreement with the results from PC577 and indicating the change has not affected the terminal emitter of the phycobiliprotein.

As the acclimating cryptophyte algae attempt to compensate for losses in light irradiance, we anticipated that the phycobiliprotein absorbance spectra would shift to mirror the regions of the restricted light spectra with the highest light intensity. Interestingly, the shoulder at $570 \mathrm{~nm}$ in PE545 did not match the region of highest light intensity transmitted through the filter, 530-540 $\mathrm{nm}$. This inconsistency may result from physical constraints; there are no other known bilins that absorb at lower wavelengths than PEB. ${ }^{26}$ The cryptophyte may also be increasing absorbance in 
regions with less light intensity to compensate, rather than shifting the overall absorbance towards the available light. Another possibility may be that the shift in PE545 absorbance plays a different role within the cellular environment. PE545 has been shown to adopt several forms within the thylakoid lumen of the cell depending on overall concentration and packing, such as being closely bound to the photosystem, arranged in stacked rods, or freely dispersed. ${ }^{27-29}$ The packing of PE545 affects how energy is funneled to chlorophylls on both PSI and PSII located in the thylakoid membrane, as energy transfer rates depend strongly on the angular distribution of PE545 relative to the chlorophylls. ${ }^{30-32}$ In photoacclimated P. sulcata, the relative amount of PE545 was much higher than that of chlorophyll, indicating that the packing arrangement and angular distribution of PE545 may be shifted to allow more efficient, vectorial energy transfer from PE545 to each photosystem. The structural confirmations of the proteins in the tightly packed environment of the cell may result in the subtle change observed in the isolated protein spectra, which are undetectable in the whole cell algae absorbance measurements. Regardless of the reason behind the unexpected absorbance shift, the reproducible change in absorbance confirms that photoacclimation has caused a change in PE545 itself.

The hallmark of a photoacclimation response is that the response is reversible; when the algae are returned to typical light conditions, protein expression reverts back to its original state within one generation. ${ }^{1}$ Another possible outcome following growth under restricted light is photoadaptation, where the restricted light conditions select for algae which contain a genomic change, resulting in a new strain of algae with a mutated version of the phycobiliprotein. Photoadaptation, therefore, stands in stark contrast to photoacclimation because the change in phycobiliprotein will not be reversible. To confirm that our observed phycobiliprotein response was reversible and thus a result of photoacclimation, we placed fresh cultures of $H$. pacifica and $P$. sulcata which had previously been grown under restricted light conditions back under full light conditions for one generation of growth. Following growth to saturation, we extracted and purified the phycobiliproteins from each culture and measured the absorbance of the phycobiliprotein. In both PC577 and PE545, we found that the absorbance spectra had reverted back to match the absorbance of phycobiliproteins grown under full light (Figure 1g,h), indicating the change in phycobiliprotein was a controllable photoacclimation response. 

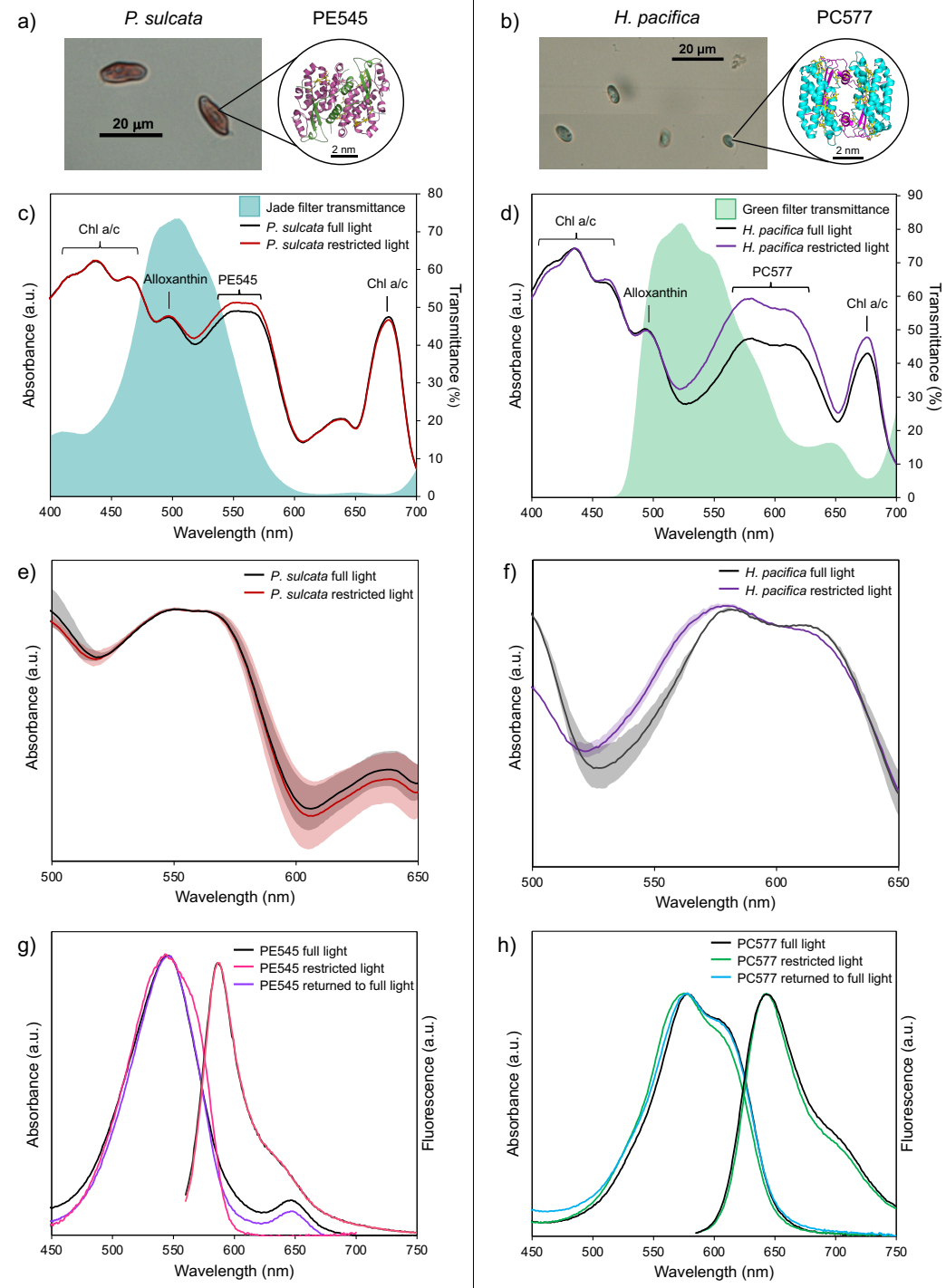

Figure 1. Microscopy images of cryptophyte algae used for the photoacclimation study, a) Proteomonas sulcata and b) Hemiselmis pacifica. Callouts show the crystal structure for the phycobiliprotein expressed in each species. The whole cell absorbance spectra, normalized to cell concentration after growth to saturation, of c) $P$. sulcata and d) $H$. pacifica grown under normal white light conditions and restricted light conditions, with labeled peaks originating from the major light harvesting protein complexes. The shaded overlays correspond to the transmittance of the filter used for light restriction. e) $P$. sulcata and f) $H$. pacifica whole cell absorbance, normalized at the major phycobiliprotein absorbance peak to illustrate shifts in the major peak wavelengths, with shaded regions showing the standard deviation in absorbance over three independent cultures. The absorbance and fluorescence spectra of the purified phycobiliproteins grown under full light, restricted light from each culture, g) PE545 and h) PC577 extracted from H. pacifica and P. sulcata, respectively. The absorbance spectra of extracted phycobiliproteins grown under restricted light and then returned to full light for one generation of growth is also shown to demonstrate reversibility. 
The changes in the absorbance peak positions and relative shoulder intensities clearly indicate that cryptophytes can modify their phycobiliproteins in response to light. This response has only been observed in one other cryptophyte species, $H$. cryptochromatica, containing the phycobiliprotein PC569. ${ }^{13}$ In this case, growth under restricted light conditions resulted in a change in relative peak intensity between the main absorbance peak and shoulder of extracted PC569, a trend that is consistent with our observations regarding the absorbance spectra shoulders of PC577 and PE545. However, this specific photoacclimation response does not yet have a clearly identified biological mechanism. We began to search for a potential mechanism by studying the relationship between protein structure and function.

Specifically, we examined two possible modifications which could occur on the protein level and influence the resultant phycobiliprotein absorbance spectra: protein structure and chromophore identity. The broad peaks of the phycobiliprotein absorbance spectra originate from eight individually bound bilin chromophores, whose electronic transitions are strongly tuned by the surrounding protein environment. The bilin chromophores are linear tetrapyrroles, structurally similar to chlorophyll but with more conformational flexibility. ${ }^{33}$ Each bilin has a site energy based on chemical character which is also influenced by the protein binding pocket and coupling to nearby bilin chromophores in the protein, resulting in broad absorbance peaks as opposed to narrow, discrete peaks for each bilin. The protein structure of phycobiliproteins is tetrameric, composed of two $\alpha$ and two $\beta$ subunits, each of which contains one and three bilin chromophores, respectively. Generally, two types of tetramer assemblies have been identified; an open confirmation, where there is a water filled gap in the center of the assembly, and a closed conformation, where there is no gap and the inner bilin chromophores are closely spaced and surrounded by the protein matrix. ${ }^{34}$

The phycobiliproteins studied here, PE545 and PC577, differ from each other in both chromophore composition and structural conformation. PE545 contains three phycoerythrobilin (PEB) chromophores on the $\beta$ subunit ( $\beta 50 / 61, \beta 82, \beta$ 158), one 15,16-dihydrobiliverdin (DBV) chromophore on the $\alpha$ subunit ( $\alpha 20$ ), and the protein assembles into a "closed" phycobiliprotein structure (Figure 1a and S2a). ${ }^{35}$ PC577, on the other hand, contains two phycocyanobilin (PCB) chromophores $(\beta 82, \beta 158)$ and one DBV $(\beta 50 / 61)$ on the $\beta$ subunits, an additional PCB on the $\alpha$ subunit ( $\alpha 19$ ), and adopts an open structure (Figure $1 \mathrm{~b}$ and S2b). ${ }^{36}$ The bilin chromophores are all attached through single cysteine linkages, except for one bilin on each $\beta$ subunit, which are attached by two cysteine linkages ( $\beta 50 / 61)$. In PE545, the DBV chromophores are the lowest in energy, and contribute to the shoulder in the absorbance spectra at $570 \mathrm{~nm}$. In contrast, the DBV chromophores in PC577 are the highest in energy and contribute to the main peak at $577 \mathrm{~nm}$, while the PCB attached at $\beta 82$ forms the lowest energy state and terminal emitter, contributing to the shoulder at $620 \mathrm{~nm} \cdot{ }^{25,33}$

The inherent dependence of the absorbance spectra on both chromophore composition and protein structure complicates the relationship between the shifts in absorbance to biological changes on the protein level. We narrowed our search by first examining whether the photoacclimated phycobiliproteins have changed in protein structure using biophysical 
measurements. Changes in protein structure will originate from changes in the apoprotein amino acid sequence, or from a post translational modification occurring after expression. Either of these changes in protein structure would result in a change to the protein mass and can be measured using electrospray ionization mass spectroscopy (ESI-MS). The mass of the $\alpha$ and $\beta$ subunits from both PE545 and PC577 did not change following growth under restricted light conditions, demonstating there is no change to amino acid sequence or post translational modifications (Table $\mathrm{S} 1$ ). We further probed for changes in apoprotein structure using CD spectroscopy, which indicates changes to the tertiary structure. We found that the CD spectra were identical for proteins from full light and restricted light conditions, indicating that no major structural change had occurred (Figure S3).

Despite the lack of evidence for a change in amino acid sequence or post translational modification, even slight perturbations to the apoprotein structure could modify the chromophore environment, resulting in subtle absorbance peaks shifts. To unequivocally determine whether the protein structure was modified following photoacclimation, we determined the X-ray crystal structure of PC577 extracted from both the native and photoacclimated proteins at 1.80 and 2.35 Å resolution, respectively (Table S2). We found that the structure for photoacclimated PC577 was identical to the native structure within experimental error, Figure 2a, with no evidence for significant conformational change or alteration in sequence. An independently-determined structure of PC577 (Dr. Kate Michie and Dr. Paul Curmi, personal communication) is essentially identical to our native crystal structure.

We specifically investigated the conformation of the PCB chromophores on the $\beta$ chain at site 82 and 158 within the crystal structure to determine whether there was any change in the geometry of the photoacclimated protein which could potentially shift in the absorbance spectra. We calculated the Fo-Fc difference maps between an atomic crystal structure model with no chromophores and the collected electron density from experiment. The Fo-Fc maps, shown as a grid for site $\beta$-158 in Figure 2 b,c, indicate where the chromophore electron density was during the x-ray measurement, which essentially represents the chromophore geometry within the protein. We overlaid a PCB ligand with the difference density map and found excellent agreement for both the native and photoacclimated PC577 crystal structures, indicating there was no change in chromophore geometry. This result, along with the consistent ESI-MS and CD results, confirm that photoacclimation does not cause a change in apoprotein structure or a modification to the PCB ligand conformation responsible for the shifting absorbance peak. We were not able to obtain high resolution x-ray diffraction data from photoacclimated PE545 protein crystals, but the ESI-MS and CD results suggest that there would be no observable change. 
a) Photoacclimated PC577

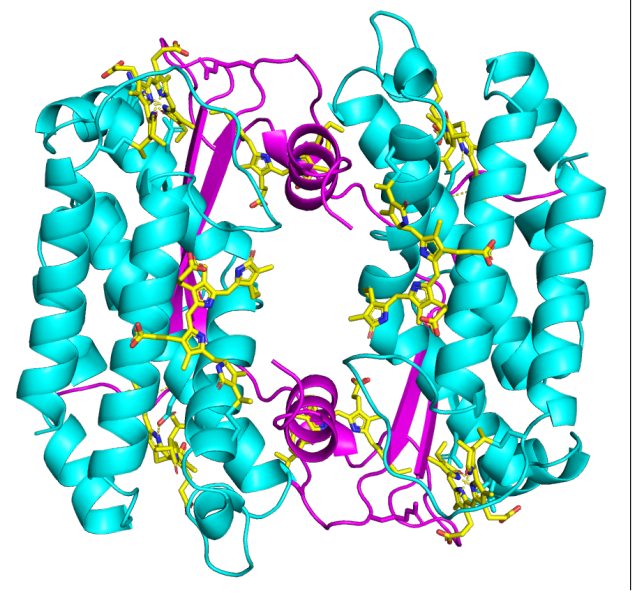

b) PCB $\beta-158$, native

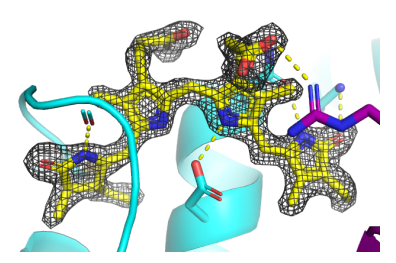

d) PCB at $\beta-158$, photoacclimated

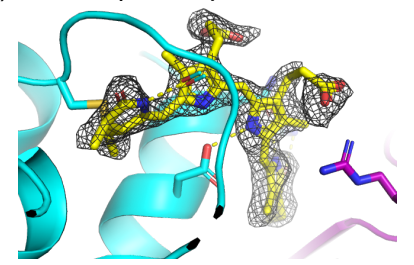

c) PCB at $\beta-158$, photoacclimated

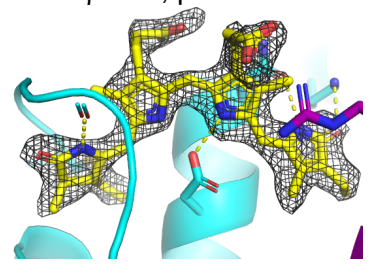

e) DBV at $\beta-158$, photoacclimated

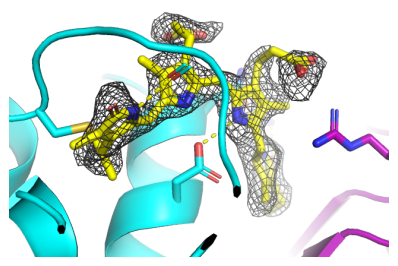

Figure 2. a) The resolved crystal structure for photoacclimated PC577. The alpha chain is in purple, the beta chain in cyan and the chromophores are shown in yellow. There is no significant difference in crystal structure from the native PC577. Residual maps calculated from the apoprotein x-ray structure without a chromophore are shown for the site $\beta-158$ in the b) native and c) photoacclimated PC577 overlaid with the PCB to demonstrate that there was no observable change in the geometry of the chromophore binding pocket following photoacclimation. The same residual map is also shown at a different angle for photoacclimated PC577 overlaid with d) a PCB chromophore and e) a DBV chromophore to show that either could fit in the active site. The DBV ligand has subtle errors in conformation of the first tetrapyrrole ring.

In the absence of a structural perturbation in the protein, the other possible change is to the chromophores. The relative contributions of each chromophore to the absorbance spectra have previously been calculated using various computational models, which we apply here to correlate changes in absorbance to specific chromophores within the phycobiliproteins. ${ }^{25,37}$ In photoacclimated PC577, we observed an increase in absorbance intensity and a shift on the higher energy side of the absorbance spectra, which correlates roughly to the contributions from the DBV chromophores, and a decrease in intensity of the shoulder at $620 \mathrm{~nm}$, which correlates roughly with the PCB chromophores. Thus, we hypothesize that PC577 synthetized during photoacclimation contain more DBV chromophores than PCB. In the case of PE545, we see an enhancement in the shoulder at $570 \mathrm{~nm}$, which again corresponds to DBV, and hypothesize that a DBV is attached at one or more of the PEB chromophore sites when P. sulcata is grown under restricted light conditions. We cannot rule out the possibility that one of the bilins has been changed to another type of tetrapyrrole bilin. Other phycobiliproteins contain alternative chromophores, such as bilin 584 or mesobiliverdin (MBV). ${ }^{26}$ However, we believe the shifts in absorbance would be more dramatic than what we observed, as the other bilins have significantly different independent site energies compared to the original chromophores. Additionally, cryptophytes have only been observed to produce one type of phycobiliprotein per species, 
indicating that the multiple pathways needed to produce alternative bilin types likely do not exist in a single cryptophyte species..$^{16,26}$

While the substitution of DBV in both photoacclimated PC577 and PE545 seems likely based on the changes to the absorbance spectra, it is difficult to determine the exact location of the PCB or PEB, respectively, that has been altered. However, we can determine which subunit contains the alternative bilin by separating, isolating, and examining the subunits of each phycobiliprotein. Following the method of Laos et al., the $\alpha$ and $\beta$ subunits were separated by lowering the $\mathrm{pH}$ of isolated protein solutions to 2 and then immediately performing HPLC to isolate the subunits from each other. Following separation, the fraction containing $\beta$ subunits were concentrated and titrated back to neutral pH (7.2 for PC577 and 6.6 for PE545). The $\beta$ subunits refolded spontaneously at neutral $\mathrm{pH}$, but will aggregate over long times ( $>4$ hours) due to the absence of $\alpha$ subunits which stabilize the structure. ${ }^{38}$ We controlled for aggregation by keeping the concentration of $\beta$ subunits within $5-10 \mu \mathrm{M}$, the concentration found previously to have the lowest amount of aggregation. We also confirmed that the $\beta$ subunits had refolded using CD measurements (Figure S4) which show consistency with the fully assembled protein and between native and photoacclimated protein $\beta$ subunits. The $\beta$ subunits from PC577 continue to show a slight blue shift and higher absorbance intensity in the region corresponding to DBV (Figure $3 b$ ). This confirms that one of the PCBs on the $\beta$ subunit has likely been modified to a DBV.

Using the PC577 crystal structures collected using x-ray diffraction, we overlaid the difference density maps with both a PCB and a DBV ligand to evaluate whether a DBV could fit the binding pocket. We found that both PCB and DBV generally agree with the difference density maps for site $\beta-158$ (Figure 2 e,f) and $\beta-82$ (Figure S5), suggesting that photoacclimated PC577 could possibly have a DBV bound at these sites, causing a shift in the absorbance spectrum. However, small changes in the stereochemistry of DBV produce a less accurate fit. We believe this discrepancy occurs from our initial guess at the stereochemistry for DBV, which was based on chemical identity. This assumption may affect the ability of our protein model to adequately represent a DBV within the apoprotein structure.

In contrast to the results for PC577, the $\beta$ subunits from PE545 did not retain the change in absorbance once separated (Figure 3a), indicating PE545 may be modified on the $\alpha$ subunit. We were unable to compare the $\alpha$ subunits at neutral $\mathrm{pH}$ because they require the presence of the $\beta$ subunits to properly fold. At $\mathrm{pH} \mathrm{2,} \mathrm{however,} \mathrm{the} \alpha$ subunit absorbance spectra are identical, suggesting there is no change to the bilin despite the lack of folded structure (Figure S6). There exists the possibility that two or more populations of $\alpha$ subunits have been incorporated into photoacclimated PE545. Recent work has identified the expression of over 20 types of PE545 $\alpha$ subunits in the cryptophyte algae Guillardia theta, which likely coexist in a pool within the cell. ${ }^{40}$ An alternative population of $\alpha$ subunits may be mixed with a larger population of native $\alpha$ subunits, complicating spectroscopic analysis. Another possible explanation for the lack of absorbance peak shift in either PE545 subunit could be that the change only manifests in the final 
tetrameric protein, where all eight chromophores are present and in the correctly folded protein environment.

a)

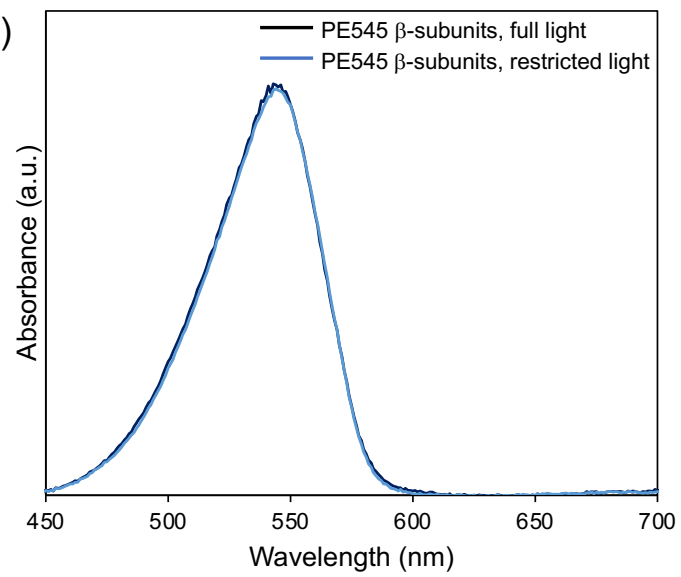

b)

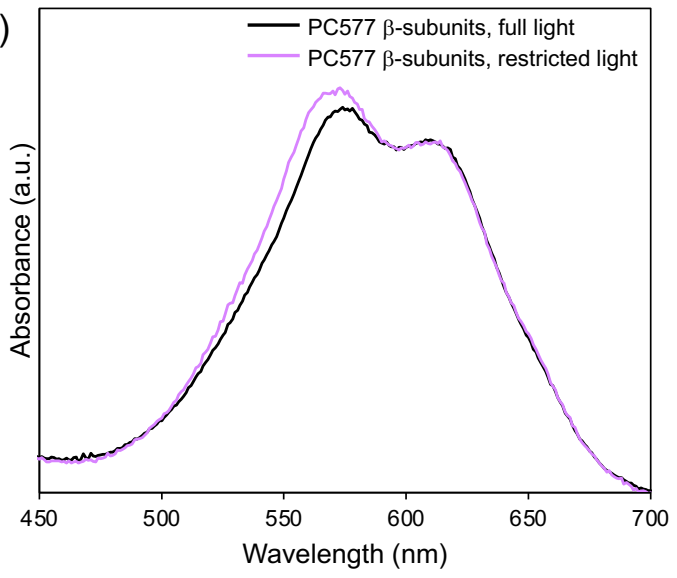

Figure 3. Absorbance spectra for $\beta$ subunits separated from a) PE545 and b) PC577 from algae grown under both full light and restricted light conditions.

We investigated whether the modified phycobiliproteins have changes in their electronic energy transfer following light absorption using transient absorption spectroscopy, which tracks the electronic state of the phycobiliprotein after a light absorption event. PC577 and PE545 have already been well characterized using transient absorption spectroscopy, ${ }^{36,41,42}$ so we only briefly summarize the major features here and then highlight major differences observed in the photoacclimated phycobiliproteins. Following light excitation at $500 \mathrm{~nm}$, both PC577 and PE545 exhibit a negative intensity ground state bleach overlapped with a stimulated emission signal, consistent with their steady-state absorbance and fluorescence signal, respectively. They also show a positive excited state absorption on both the blue edge and the red edge, typically at wavelengths $<500 \mathrm{~nm}$ and $>660 \mathrm{~nm}$. At short times $(<1 \mathrm{ps}$ ), there is a transfer of intensity in the negative signal from a broad ground state bleach to the stimulated emission signal, which indicates a transfer of energy from the higher lying bilin energy states to the lower energy states. In PC577, this occurs 
from the DBVs to the PCBs; in PE545, this occurs from the PEBs to the relatively lower lying DBVs.

Based on the previously presented fluorescence spectra (Figure $1 \mathrm{~g}, \mathrm{~h}$ ), we did not anticipate a major change to the stimulated emission signal originating from the lowest lying energy states. However, we did anticipate a change in ground state bleach feature of the transient absorbance spectra taken at early times as this correlates to the steady state absorbance spectra. The spectral trace of the fully assembled PC577 at $250 \mathrm{fs,} \mathrm{Figure} \mathrm{4a,} \mathrm{shows} \mathrm{a} \mathrm{similar} \mathrm{blue} \mathrm{shift} \mathrm{in} \mathrm{the} \mathrm{ground}$ state bleach signal for the proteins grown under restricted light conditions. The same shift is also observed in the transient absorbance spectra of PC577 isolated $\beta$ subunit, Figure $4 \mathrm{~b}$. We examined the transfer of energy from the initial ground state bleach to the stimulated emission population by comparing the decay kinetics of the peak at $577 \mathrm{~nm}$ and $630 \mathrm{~nm}$, which additionally correspond to the peaks which were modified in the photoacclimated proteins. We found no significant change to the kinetics in either the fully assembled or isolated $\beta$ subunits of PC577 following photoacclimation. This result is perhaps unsurprising; previous studies have demonstrated the strong role played by the protein environment on energy transfer. ${ }^{37,41,43,44}$ As we found no change in protein structure, we did not expect to see a major change in energy transfer kinetics. 

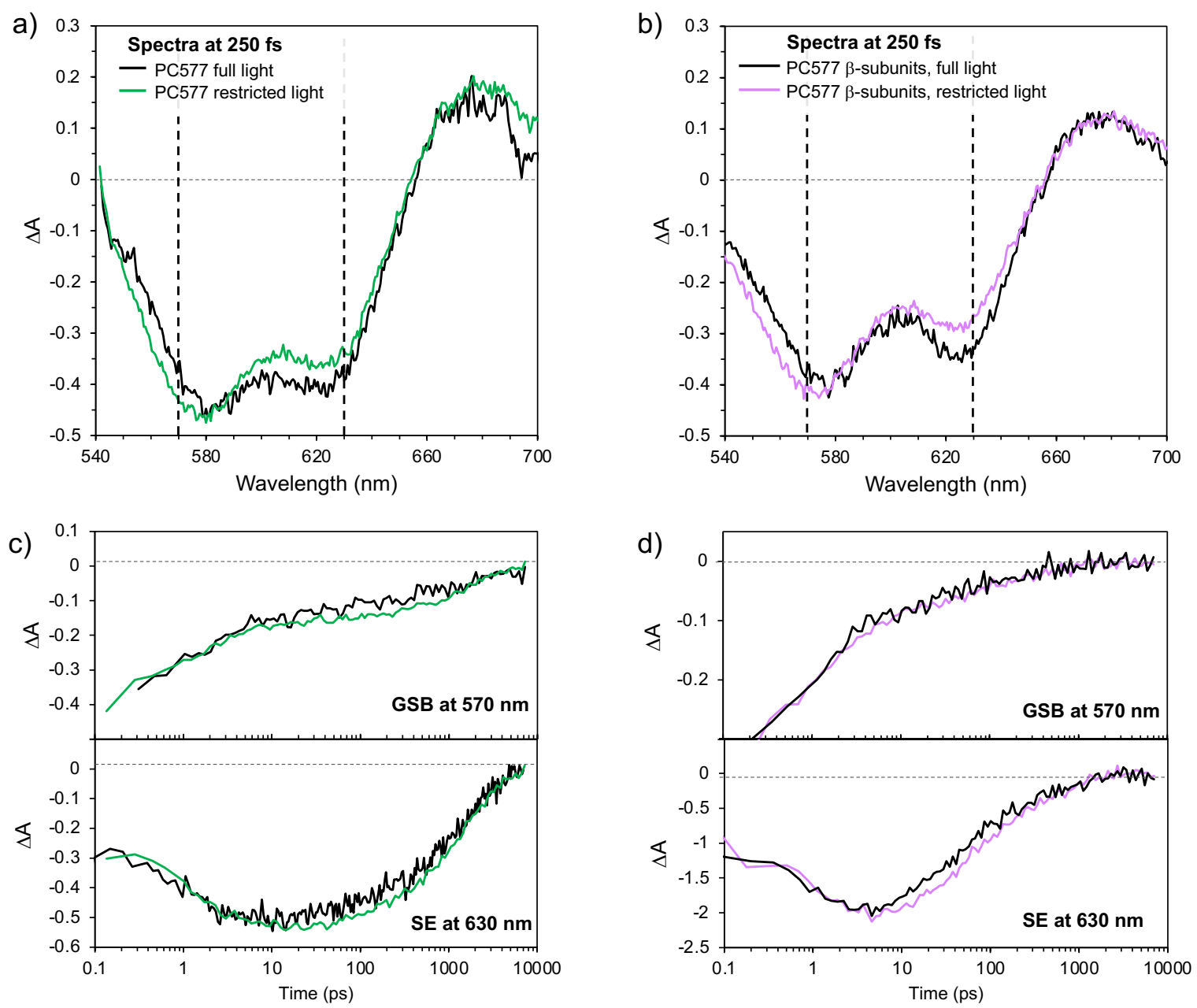

Figure 4. Spectral slices taken from transient absorption measures on native and photoacclimated PC577, a) fully assembled and b) isolated $\beta$ subunits. Kinetic traces (c,d) show no change to decay kinetics in the photoacclimated proteins.

Similarly to PC577, the spectral traces of assembled PE545 taken at 500 fs show the preservation of an enhanced absorbance shoulder at $590 \mathrm{~nm}$, Figure 5a, matching the change observed in the steady state absorbance. The energy transfer kinetics of assembled PE545 also appear to be the same in both the native and photoacclimated proteins, Figure $5 \mathrm{c}$. However, the isolated $\beta$ subunits from PE545 show strikingly different kinetics in the photoacclimated case. This result was surprising, as the spectral slices at 500 fs of the $\beta$ subunit populations look identical (Figure 5b), no longer showing an enhanced GSB around $590 \mathrm{~nm}$ at early times which was observed in the fully assembled protein. However, the kinetic traces are very different: the $\beta$ subunits taken from photoacclimated PE545 have longer lifetimes at both $555 \mathrm{~nm}$ and $570 \mathrm{~nm}$, Figure 5d, indicating a slower transfer of energy from higher to lower energy states during the first $50 \mathrm{ps}$. To ensure the energy transfer was not unintentionally changed due to protein structure, we took CD measurements of the PE545 $\beta$ subunits immediately before and after the TA 
measurements to ensure the subunits remained properly folded (Figure S5), and found the CD spectra to be consistent with the fully assembled PE545 (Figure S2). In the absence of a structural change, we consider the case where one of the PEBs on the $\beta$ subunit has been switched for a DBV resulting in a lower site energy for the chromophore position. This alteration should result in a change to the energy transfer within the $\beta$ subunit as the energy landscape is no longer defined by three degenerate PEB chromophores. The change in energy transfer was less noticeable in the assembled PE545, likely due to the presence of the $\alpha$ subunits that contain the previously identified terminal emitter of the protein, DBV, and will dominate the overall energy transfer pathways. ${ }^{33}$
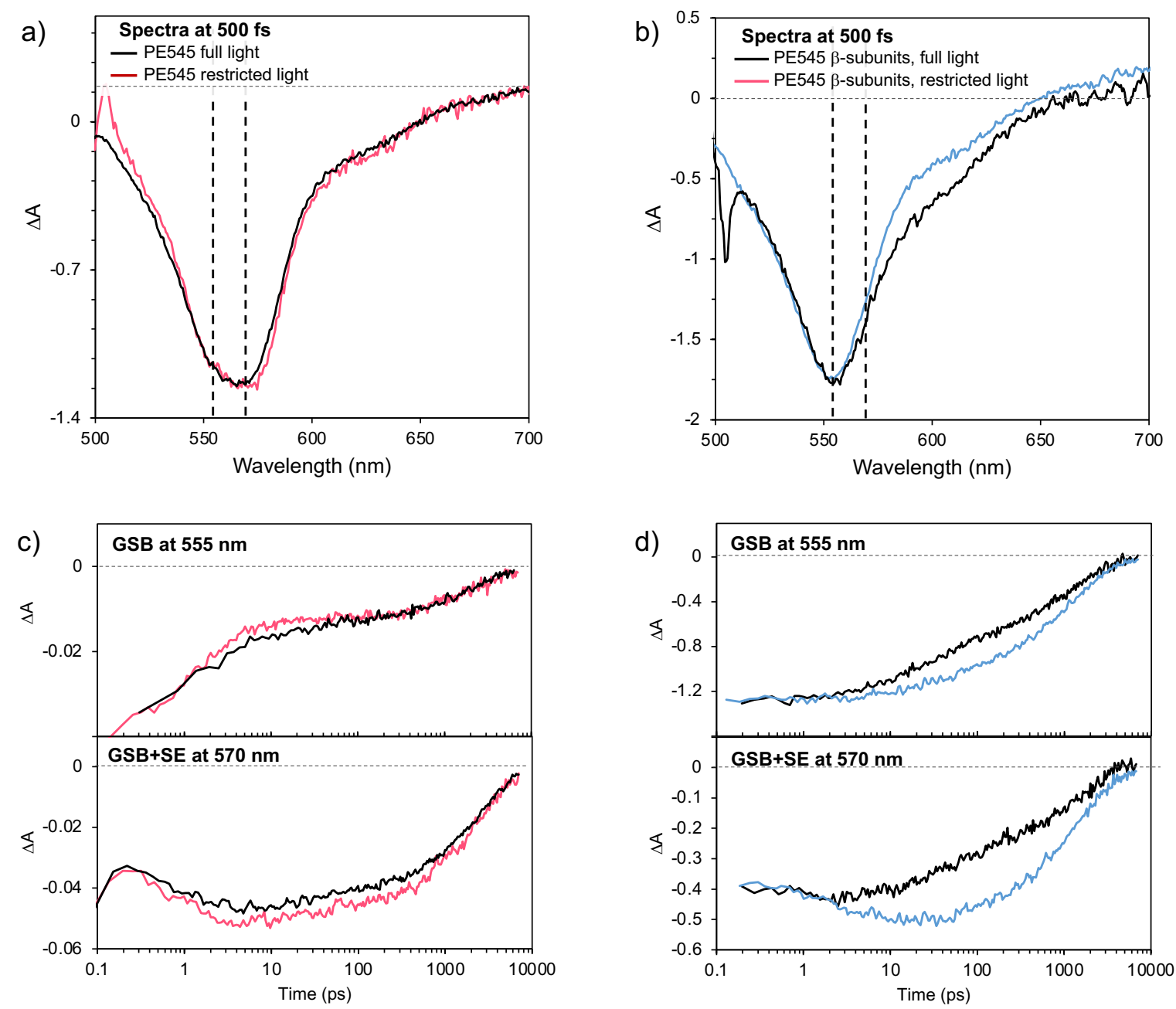

Figure 5. Spectral slices taken from transient absorption measures on native and photoacclimated PE545, a) fully assembled and b) isolated $\beta$ subunits. Kinetic traces show no change to decay kinetics in the assembled photoacclimated proteins (c), but significant changes in the isolated $\beta$ subunits (d). 
Surprisingly, the overall energy transfer dynamics in PC577 and PE545 were not significantly affected by photoacclimation. We believe that this result shows the importance of the protein structure in guiding the energy transfer between bilins following the capture of light, adding further evidence to previous findings which also show the influence of the protein structure on energy transfer. ${ }^{37,41,43,44}$ Here, we have shown the robust nature of energy transfer within the phycobiliprotein, even after a change in bilin identity.

The original question of this study was how cryptophyte algae are capable of photoacclimating to changes in spectral quality if they lack a phycobilisome. We have found that cryptophytes are capable incorporating alternative bilins onto their individual phycobiliproteins to compensate for changes in the available light spectrum. The mechanism behind this new photoacclimation pathway is likely the result of the biosynthetic pathways used by cryptophyte algae to produce and attach individual bilin chromophores to the apoprotein structure during phycobiliprotein synthesis. While these pathways are not yet fully elucidated for the species studied here, the cryptophyte algae Guillardia theta was recently shown to attach PEB chromophores with the same enzymes used by cyanobacteria, phycobilin lyases. ${ }^{39}$ The cyanobacteria Synechococcus possesses a photoacclimation process known as differential chromophorylation, where individual bilin chromophores on the phycobilisome are replaced though cellular regulation of phycobilin lyases within the cell. ${ }^{18}$ Cryptophytes are distant relatives of cyanobacteria, and inherited their phycobiliprotein $\beta$ subunit synthesis pathways. ${ }^{45}$ Based on the similarities of our observed photoacclimation response and the differential chromophorylation mechanism observed in cyanobacteria, the biosynthetic pathways leading to photoacclimation appear to be evolutionarily linked.

\section{Conclusion}

Herein, we have demonstrated that two cryptophyte algae strains $P$. sulcata and H. pacifica are capable of photoacclimation when grown under spectrally restricted light conditions. Both species undergo photoacclimation by increasing the overall concentration of phycobiliproteins and through the modification of their phycobiliprotein absorbance spectra. In depth analysis of the phycobiliproteins found no change to protein sequence nor structure. Thus, we concluded that photoacclimation was achieved by changing one or more of the tetrapyrrole bilin chromophores bound to the protein. The change to H. pacifica's phycobiliprotein, PC577, was confirmed to be associated with the $\beta$ subunit because the absorbance spectra shift was preserved in the isolated $\beta$ subunit population. The change to $P$. sulcata's phycobiliprotein, PE545, was more subtle but still concluded to be located on the $\beta$ subunits based on the observation of modified transient absorption kinetics in the isolated $\beta$ subunits. We suggest that the photoacclimation mechanism likely involves control of the identity of bilins attached to the $\beta$ subunits during protein synthesis because modification of the chromophores in the folded protein is unlikely. ${ }^{39}$ This research sheds light on a newly identified cryptophyte photoacclimation processes, and provides a key insight into how natural systems regulate photosynthesis using pre-existing biosynthetic pathways. 


\section{Supporting Information Description}

Additional information regarding experimental methods, protein spectroscopy and X-ray data are available in the supporting information. The SI includes algae growth curves, CD spectra, absorbance spectra of purified $\alpha$ subunits, ESI-MS results, and X-ray data collection and structure refinement statistics.

\section{Acknowledgements}

The authors thank Dr. Beverley Green of the Department of Biology at The University of British Columbia for helpful discussion and experimental insights. We also thank Dr. Paul Curmi of the University of New South Wales for useful protein crystallization discussions and for allowing us to compare our native PC577 structure to their previously resolved PC577 crystal structure. The authors wish to thank Dr. Michael Hecht's lab in the Chemistry Department of Princeton University for use of their HPLC. Mass spectrometry and CD measurements were performed in the Mass Spectrometry and Biophysics core facility, respectively, in the Department of Chemistry at Princeton University. The protein crystallography measurements were obtained in the Protein Crystallography Core of the Molecular Biology Department at Princeton University. This work was funded by the Princeton University Writing Center and CIFAR (Canadian Institute for Advanced Research). Dr. Gregory Scholes is CIFAR fellow. 


\section{References}

(1) Falkowski, P. G.; Chen, Y.-B. Photoacclimation of Light Harvesting Systems in Eukaryotic Algae. In Light-Harvsting Antennas in Photosynthesis; Advances in Photosynthesis and Respiration; Kluwer Academic Publishers, 2003; Vol. 13, pp 423-447.

(2) Grossman, A. R.; Schaefer, M. R.; Chiang, G. G.; Collier, J. L. The Phycobilisome, a LightHarvesting Complex Responsive to Environmental Conditions. Microbiol. Rev. 1993, 57 (3), 725-749.

(3) Watanabe, M.; Ikeuchi, M. Phycobilisome: Architecture of a Light-Harvesting Supercomplex. Photosynth. Res. 2013, 116 (2), 265-276. https://doi.org/10.1007/s11120013-9905-3.

(4) Tandeau de Marsac, N. Occurrence and Nature of Chromatic Adaptation in Cyanobacteria. J. Bacteriol. 1977, 130 (1), 82-91. https://doi.org/10.1128/JB.130.1.82-91.1977.

(5) Gaiducov, N. Uber Den Einfluss Farbigen Lichtes Auf Die Farbung Lebender Oscillarien. Abh. K. Akad. Wiss. 1902, 5, 1-36.

(6) Stomp, M.; van Dijk, M. A.; van Overzee, H. M. J.; Wortel, M. T.; Sigon, C. A. M.; Egas, M.; Hoogveld, H.; Gons, H. J.; Huisman, J. The Timescale of Phenotypic Plasticity and Its Impact on Competition in Fluctuating Environments. Am. Nat. 2008, 172 (5), E169-E185. https://doi.org/10.1086/591680.

(7) Gan, F.; Zhang, S.; Rockwell, N. C.; Martin, S. S.; Lagarias, J. C.; Bryant, D. A. Extensive Remodeling of a Cyanobacterial Photosynthetic Apparatus in Far-Red Light. Science 2014, 345 (6202), 1312-1317. https://doi.org/10.1126/science.1256963.

(8) Grébert, T.; Doré, H.; Partensky, F.; Farrant, G. K.; Boss, E. S.; Picheral, M.; Guidi, L.; Pesant, S.; Scanlan, D. J.; Wincker, P.; Acinas, S. G.; Kehoe, D. M.; Garczarek, L. Light Color Acclimation Is a Key Process in the Global Ocean Distribution of Synechococcus Cyanobacteria. Proc. Natl. Acad. Sci. 2018, 115 (9), E2010-E2019. https://doi.org/10.1073/pnas.1717069115.

(9) Flombaum, P.; Gallegos, J. L.; Gordillo, R. A.; Rincon, J.; Zabala, L. L.; Jiao, N.; Karl, D. M.; Li, W. K. W.; Lomas, M. W.; Veneziano, D.; Vera, C. S.; Vrugt, J. A.; Martiny, A. C. Present and Future Global Distributions of the Marine Cyanobacteria Prochlorococcus and Synechococcus. Proc. Natl. Acad. Sci. 2013, 110 (24), 9824-9829.

https://doi.org/10.1073/pnas.1307701110.

(10) Sanfilippo, J. E.; Garczarek, L.; Partensky, F.; Kehoe, D. M. Chromatic Acclimation in Cyanobacteria: A Diverse and Widespread Process for Optimizing Photosynthesis. Annu. Rev. Microbiol. 2019, 73 (1), 407-433. https://doi.org/10.1146/annurev-micro-020518115738.

(11) Kehoe, D. M. Chromatic Adaptation and the Evolution of Light Color Sensing in Cyanobacteria. Proc. Natl. Acad. Sci. 2010, 107 (20), 9029-9030. https://doi.org/10.1073/pnas.1004510107.

(12) Gutu, A.; Kehoe, D. M. Emerging Perspectives on the Mechanisms, Regulation, and Distribution of Light Color Acclimation in Cyanobacteria. Mol. Plant 2012, 5 (1), 1-13. https://doi.org/10.1093/mp/ssr054.

(13) Heidenreich, K. M.; Richardson, T. L. Photopigment, Absorption, and Growth Responses of Marine Cryptophytes to Varying Spectral Irradiance. J. Phycol. 2019, 56 (2), 507-520. https://doi.org/10.1111/jpy.12962. 
(14) Wehrmeyer, W. Phycobiliproteins and Phycobiliprotein Organization in the Photosynthetic Apparatus of Cyanobacteria, Red Algae, and Cryptophytes. In Proteins and Nucleic Acids in Plant Systematics; Jensen, U., Fairbrothers, D. E., Eds.; Proceedings in Life Sciences; Springer: Berlin, Heidelberg, 1983; pp 143-167. https://doi.org/10.1007/978-3-64269287-1_10.

(15) Green, B. R. What Happened to the Phycobilisome? Biomolecules 2019, 9 (11), 748. https://doi.org/10.3390/biom9110748.

(16) MacColl, R. Phycobiliproteins; CRC Press, 2018. https://doi.org/10.1201/9781351075565.

(17) Cunningham, B. R.; Greenwold, M. J.; Lachenmyer, E. M.; Heidenreich, K. M.; Davis, A. C.; Dudycha, J. L.; Richardson, T. L. Light Capture and Pigment Diversity in Marine and Freshwater Cryptophytes. J. Phycol. 2019, 55 (3), 552-564.

https://doi.org/10.1111/jpy.12816.

(18) Everroad, C.; Six, C.; Partensky, F.; Thomas, J.-C.; Holtzendorff, J.; Wood, A. M. Biochemical Bases of Type IV Chromatic Adaptation in Marine Synechococcus Spp. J. Bacteriol. 2006, 188 (9), 3345-3356. https://doi.org/10.1128/JB.188.9.3345-3356.2006.

(19) Shukla, A.; Biswas, A.; Blot, N.; Partensky, F.; Karty, J. A.; Hammad, L. A.; Garczarek, L.; Gutu, A.; Schluchter, W. M.; Kehoe, D. M. Phycoerythrin-Specific Bilin Lyase-Isomerase Controls Blue-Green Chromatic Acclimation in Marine Synechococcus. Proc. Natl. Acad. Sci. 2012, 109 (49), 20136-20141. https://doi.org/10.1073/pnas.1211777109.

(20) Sanfilippo, J. E.; Nguyen, A. A.; Karty, J. A.; Shukla, A.; Schluchter, W. M.; Garczarek, L.; Partensky, F.; Kehoe, D. M. Self-Regulating Genomic Island Encoding Tandem Regulators Confers Chromatic Acclimation to Marine Synechococcus. Proc. Natl. Acad. Sci. 2016, 113 (21), 6077-6082. https://doi.org/10.1073/pnas.1600625113.

(21) Takaichi, S. Carotenoids in Algae: Distributions, Biosyntheses and Functions. Mar. Drugs 2011, 9 (6), 1101-1118. https://doi.org/10.3390/md9061101.

(22) Falkowski, P. G.; Raven, J. A. Aquatic Photosynthesis, 2nd ed.; Princeton University Press: Princeton, 2007.

(23) Scholes, G. D.; Mirkovic, T.; Turner, D. B.; Fassioli, F.; Buchleitner, A. Solar Light Harvesting by Energy Transfer: From Ecology to Coherence. Energy Environ. Sci. 2012, 5 (11), 9374-9393. https://doi.org/10.1039/C2EE23013E.

(24) Kaňa, R.; Kotabová, E.; Sobotka, R.; Prášil, O. Non-Photochemical Quenching in Cryptophyte Alga Rhodomonas Salina Is Located in Chlorophyll a/c Antennae. PLOS ONE 2012, 7 (1), e29700. https://doi.org/10.1371/journal.pone.0029700.

(25) Corbella, M.; Cupellini, L.; Lipparini, F.; Scholes, G. D.; Curutchet, C. Spectral Variability in Phycocyanin Cryptophyte Antenna Complexes Is Controlled by Changes in the APolypeptide Chains. ChemPhotoChem 2019, 3 (9), 945-956. https://doi.org/10.1002/cptc.201900045.

(26) Wedemayer, G. J.; Kidd, D. G.; Glazer, A. N. Cryptomonad Biliproteins: Bilin Types and Locations. Photosynth. Res. 1996, 48 (1-2), 163-170.

https://doi.org/10.1007/BF00041006.

(27) Ludwig, M.; Gibbs, S. P. Localization of Phycoerythrin at the Lumenal Surface of the Thylakoid Membrane in Rhodomonas Lens. J. Cell Biol. 1989, 108 (3), 875-884. https://doi.org/10.1083/jcb.108.3.875.

(28) Spear-bernstein, L.; Miller, K. R. Unique Location of the Phycobiliprotein Light-Harvesting Pigment in the Cryptophyceae. J. Phycol. 1989, 25 (3), 412-419.

https://doi.org/10.1111/j.1529-8817.1989.tb00245.x. 
(29) Vesk, M.; Dwarte, D.; Fowler, S.; Hiller, R. G. Freeze Fracture Immunocytochemistry of Light-Harvesting Pigment Complexes in a Cryptophyte. Protoplasma 1992, 170 (3-4), 166-176. https://doi.org/10.1007/BF01378791.

(30) van der Weij-De Wit, C. D.; Doust, A. B.; van Stokkum, I. H. M.; Dekker, J. P.; Wilk, K. E.; Curmi, P. M. G.; Scholes, G. D.; van Grondelle, R. How Energy Funnels from the Phycoerythrin Antenna Complex to Photosystem I and Photosystem II in Cryptophyte Rhodomonas CS24 Cells. J. Phys. Chem. B 2006, 110 (49), 25066-25073. https://doi.org/10.1021/jp061546w.

(31) Stadnichuk, I. N.; Novikova, T. M.; Miniuk, G. S.; Boichenko, V. A.; Bolychevtseva, Yu. V.; Gusev, E. S.; Lukashev, E. P. Phycoerythrin Association with Photosystem II in the Cryptophyte Alga Rhodomonas Salina. Biochem. Mosc. 2020, 85 (6), 679-688. https://doi.org/10.1134/S000629792006005X.

(32) Mirkovic, T.; Wilk, K. E.; Curmi, P. M. G.; Scholes, G. D. Phycobiliprotein Diffusion in Chloroplasts of Cryptophyte Rhodomonas CS24. Photosynth. Res. 2009, 100 (1), 7-17. https://doi.org/10.1007/s11120-009-9412-8.

(33) Curutchet, C.; Novoderezhkin, V. I.; Kongsted, J.; Muñoz-Losa, A.; van Grondelle, R.;

Scholes, G. D.; Mennucci, B. Energy Flow in the Cryptophyte PE545 Antenna Is Directed by Bilin Pigment Conformation. J. Phys. Chem. B 2013, 117 (16), 4263-4273. https://doi.org/10.1021/jp305033d.

(34) Harrop, S. J.; Wilk, K. E.; Dinshaw, R.; Collini, E.; Mirkovic, T.; Teng, C. Y.; Oblinsky, D. G.; Green, B. R.; Hoef-Emden, K.; Hiller, R. G.; Scholes, G. D.; Curmi, P. M. G. SingleResidue Insertion Switches the Quaternary Structure and Exciton States of Cryptophyte Light-Harvesting Proteins. Proc. Natl. Acad. Sci. 2014, 111 (26), E2666-E2675. https://doi.org/10.1073/pnas.1402538111.

(35) Wilk, K. E.; Harrop, S. J.; Jankova, L.; Edler, D.; Keenan, G.; Sharples, F.; Hiller, R. G.; Curmi, P. M. G. Evolution of a Light-Harvesting Protein by Addition of New Subunits and Rearrangement of Conserved Elements: Crystal Structure of a Cryptophyte Phycoerythrin at 1.63-A Resolution. Proc. Natl. Acad. Sci. 1999, 96 (16), 8901-8906. https://doi.org/10.1073/pnas.96.16.8901.

(36) McClure, S. D.; Turner, D. B.; Arpin, P. C.; Mirkovic, T.; Scholes, G. D. Coherent Oscillations in the PC577 Cryptophyte Antenna Occur in the Excited Electronic State. $J$. Phys. Chem. B 2014, 118 (5), 1296-1308. https://doi.org/10.1021/jp411924c.

(37) Curutchet, C.; Kongsted, J.; Muñoz-Losa, A.; Hossein-Nejad, H.; Scholes, G. D.;

Mennucci, B. Photosynthetic Light-Harvesting Is Tuned by the Heterogeneous Polarizable Environment of the Protein. J. Am. Chem. Soc. 2011, 133 (9), 3078-3084. https://doi.org/10.1021/ja110053y.

(38) Laos, A. J.; Dean, J. C.; Toa, Z. S. D.; Wilk, K. E.; Scholes, G. D.; Curmi, P. M. G.; Thordarson, P. Cooperative Subunit Refolding of a Light-Harvesting Protein through a Self-Chaperone Mechanism. Angew. Chem. 2017, 129 (29), 8504-8508. https://doi.org/10.1002/ange.201607921.

(39) Overkamp, K. E.; Gasper, R.; Kock, K.; Herrmann, C.; Hofmann, E.; Frankenberg-Dinkel, N. Insights into the Biosynthesis and Assembly of Cryptophycean Phycobiliproteins. $J$. Biol. Chem. 2014, 289 (39), 26691-26707. https://doi.org/10.1074/jbc.M114.591131.

(40) Kieselbach, T.; Cheregi, O.; Green, B. R.; Funk, C. Proteomic Analysis of the Phycobiliprotein Antenna of the Cryptophyte Alga Guillardia Theta Cultured under 
Different Light Intensities. Photosynth. Res. 2018, 135 (1-3), 149-163. https://doi.org/10.1007/s11120-017-0400-0.

(41) Jumper, C. C.; van Stokkum, I. H. M.; Mirkovic, T.; Scholes, G. D. Vibronic Wavepackets and Energy Transfer in Cryptophyte Light-Harvesting Complexes. J. Phys. Chem. B 2018, 122 (24), 6328-6340. https://doi.org/10.1021/acs.jpcb.8b02629.

(42) Doust, A. B.; van Stokkum, I. H. M.; Larsen, D. S.; Wilk, K. E.; Curmi, P. M. G.; van Grondelle, R.; Scholes, G. D. Mediation of Ultrafast Light-Harvesting by a Central Dimer in Phycoerythrin 545 Studied by Transient Absorption and Global Analysis. J. Phys. Chem. B 2005, 109 (29), 14219-14226. https://doi.org/10.1021/jp051173j.

(43) Collini, E.; Wong, C. Y.; Wilk, K. E.; Curmi, P. M. G.; Brumer, P.; Scholes, G. D. Coherently Wired Light-Harvesting in Photosynthetic Marine Algae at Ambient Temperature. Nature 2010, 463 (7281), 644-647. https://doi.org/10.1038/nature08811.

(44) Dean, J. C.; Mirkovic, T.; Toa, Z. S. D.; Oblinsky, D. G.; Scholes, G. D. Vibronic Enhancement of Algae Light Harvesting. Chem 2016, 1 (6), 858-872. https://doi.org/10.1016/j.chempr.2016.11.002.

(45) Light-Harvesting Antennas in Photosynthesis; Green, B. R., Parson, W. W., Eds.; Advances in photosynthesis and respiration; Kluwer Academic: Dordrecht; Boston, 2003. 